\title{
A two-cocycle on the group of symplectic diffeomorphisms
}

\author{
Światosław Gal · Jarek Kędra
}

Received: 25 October 2010 / Accepted: 14 April 2011 / Published online: 13 May 2011

(C) The Author(s) 2011. This article is published with open access at Springerlink.com

\begin{abstract}
We investigate the properties of a two-cocycle on the group of symplectic diffeomorphisms of an exact symplectic manifold defined by Ismagilov, Losik, and Michor. We provide both vanishing and nonvanishing results and applications to foliated symplectic bundles and to Hamiltonian actions of finitely generated groups.
\end{abstract}

Keywords Symplectic manifold $\cdot$ Kähler cocycle $\cdot$ Foliation

Mathematics Subject Classification (2010) $\quad$ 53D05 $\cdot$ 57S25

\section{Introduction}

Let $(M, d \lambda)$ be a connected exact symplectic manifold with trivial first real cohomology, $H^{1}(M ; \mathbf{R})=0$. In this paper we investigate a two-cocycle $\mathfrak{G}$ on the group $\operatorname{Symp}(M, d \lambda)$ of symplectic diffeomorphisms of $(M, d \lambda)$. This cocycle was defined by Ismagilov et al. [5]

Ś. Gal was partially supported by Polish MNiSW grant N N201 541738 and Swiss NSF Sinergia Grant CRSI22-130435.

Ś. Gal (凶)

Instytut Matematyczny, Uniwersytet Wrocławski, pl. Grunwaldzki 2/4, 50-384 Wrocław, Poland e-mail:sgal@math.uni.wroc.pl

S. Gal

Fakultät für Mathematik, Universität Wien, Nordbergstrasse 15, 1090 Wien, Austria

J. Kędra

Mathematical Sciences, University of Aberdeen, Fraser Noble Building,

Aberdeen, AB243 UE, Scotland, UK

e-mail: kedra@abdn.ac.uk

J. Kędra

Institute of Mathematics, University of Szczecin, Wielkopolska 15, 70-451 Szczecin, Poland 
where they proved that it is cohomologically nontrivial when $M$ is either the standard symplectic $\mathbf{R}^{2 n}$ or a Hermitian symmetric space. The following theorem generalises their results.

Theorem 1.1 Let $(M, d \lambda)$ be the universal cover of a closed symplectic manifold $(X, \sigma)$. The cocycle $\mathfrak{G}$ represents a nonzero cohomology class.

A symplectic manifold $(X, \sigma)$ whose universal cover is exact is called symplectically aspherical. The reason is that the property can be equivalently characterised by the vanishing of the symplectic area of every sphere in $X$. More precisely, $(X, \sigma)$ is symplectically aspherical if and only if

$$
\int_{S^{2}} s^{*} \sigma=0
$$

for every smooth map $s: S^{2} \rightarrow X$ (see [7] for a survey).

1.A. Vanishing properties It is interesting to ask about the restriction of the cocycle $\mathfrak{G}$ to various subgroups of the group symplectic diffeomorphisms of $(M, d \lambda)$. It turns out that its cohomology class vanishes on the subgroup of compactly supported symplectic diffeomorphisms $\operatorname{Symp}_{c}(M, d \lambda)$ and on subgroups preserving certain isotropic submanifolds.

Theorem 1.2 The cocycle $\mathfrak{G}$ restricted to $\operatorname{Symp}_{c}(M, d \lambda)$ represents the trivial cohomology class.

A subset $L \subset M$ is isotropic if $i^{*} \lambda$, where $i: L \rightarrow M$ is the inclusion map, is a closed one-form. We say that isotropic submanifold $L \subset M$ is exact isotropic if $i^{*} \lambda$ is exact. This is to say that $\left[i^{*} \lambda\right]=0$ in $H^{1}(L ; \mathbf{R})$. It is always the case if $b_{1}(L)=0$.

Let $\operatorname{Symp}_{L}(M, d \lambda):=\{f \in \operatorname{Symp}(M, d \lambda) \mid f(L)=L\}$ be the group of symplectic diffeomorphisms preserving the submanifold $L$.

Theorem 1.3 Let $i: L \rightarrow M$ be the inclusion of a closed connected exact isotropic submanifold. Then $\mathfrak{G}$ restricted to the group $\operatorname{Symp}_{L}(M, d \lambda)$ represents the trivial cohomology class.

If $(M, d \lambda)$ is the universal cover of a closed symplectic manifold $(X, \sigma)$ then the group $\operatorname{Ham}(X, \sigma)$ can be viewed as a subgroup of $\operatorname{Symp}(M, d \lambda)$.

Theorem 1.4 The cocycle $\mathfrak{G}$ restricted to $\operatorname{Ham}(X . \sigma)$ represents the trivial cohomology class.

1.B. Hermitian symmetric spaces If $M$ is a Hermitian symmetric space of noncompact type (see [4] or [17, Chapter 3] for definitions) then the connected component $G:=\operatorname{Isom}^{\circ}(M)$ of the group of the isometries of the Kähler metric admits a nontrivial bounded two-cocycle $\mathfrak{K}$ called the Kähler cocycle. It is defined by the integration of the Kähler form over geodesic trilaterals. More precisely, fix a reference point $x \in M$ and define

$$
\mathfrak{K}(g, h):=\int_{\triangle} \sigma,
$$

where $\triangle \subset M$ is a geodesic trilateral with vertices $x, g(x), g h(x)$ and $\sigma \in \omega^{2}(M)$ is the Kähler form.

It is known [17, Section 5.2] that the Kähler cocycle is bounded. If $\Gamma \subset G$ is a uniform lattice (i.e. a discrete subgroup such that the quotient $\Gamma \backslash G$ is compact) then the pull back of 
the Kähler cocycle represents a nontrivial cohomology class in $H^{2}(\Gamma ; \mathbf{R})$. This class is equal to the class represented by the Kähler form of the compact orbifold $X=\Gamma \backslash M$. A detailed presentation can be found in Wienhard [17, Chapter 5].

The following result was proved in [5, Theorem 5.1].

Theorem 1.5 Let $(M, d \lambda)$ be a Hermitian symmetric space of noncompact type and let $G \subset \operatorname{Symp}(M, d \lambda)$ be the connected component of the group of isometries of the Kähler metric. Then the pullback of the cocycle $\mathfrak{G}$ to $G$ is cohomologous to the Kähler cocycle $\mathfrak{K}$.

In Proposition 3.5, we observe that, under suitable choices made, the cocycles $\mathfrak{G}$ and $\mathfrak{K}$ are in fact equal.

1.C. Boundedness properties Theorem 1.5 shows that the restriction of $\mathfrak{G}$ to a certain subgroup is a bounded two-cocycle. This is not the case in general.

Theorem 1.6 The two-cocycle $\mathfrak{G}$ is unbounded on $\operatorname{Symp}(M, d \lambda)$. Moreover, if $(M, d \lambda)$ is the universal cover of a closed symplectic manifold $(X, \sigma)$ then the restriction of $\mathfrak{G}$ to $\operatorname{Ham}(X, \sigma)$ is unbounded.

We investigate boundedness properties of $\mathfrak{G}$ in Sect. 4. We then apply these properties to prove a theorem of Polterovich about Hamiltonian actions of finitely generated groups on symplectically hyperbolic manifolds. This and other applications are presented in Sect. 5 .

\section{Definition of the cocycle}

We refer the reader to [1] for the standard definitions and facts about cohomology of groups. In particular, we would make use of the fact that group cohomology is the same as the cohomology of the classifying space of the group when the group is endowed with discrete topology [1, Section I.4]. A topological group $G$ considered with the discrete topology will be denoted $G^{d}$.

Let $(M, d \lambda)$ be an exact symplectic manifold with $H^{1}(M ; \mathbf{R})=0$. If $g: M \rightarrow M$ is a symplectic diffeomorphism then the one-form $g^{*}(\lambda)-\lambda$ is closed. Thus the integral $\int_{\ell} g^{*}(\lambda)-\lambda$ depends only on the endpoints of the path $\ell:[0,1] \rightarrow M$. In what follows we shall denote this integral by

$$
\int_{x}^{y} g^{*}(\lambda)-\lambda
$$

where $x=\ell(0)$ and $y=\ell(1)$.

Let $x \in M$ be a reference point. Following Ismagilov, Losik and Michor [5], we define a two-cocycle $\mathfrak{G}_{x, \lambda}$ on the discrete group $\operatorname{Symp}(M, d \lambda)$ of symplectic diffeomorphisms of $(M, d \lambda)$ by

$$
\mathfrak{G}_{x, \lambda}(g, h):=\int_{x}^{h(x)} g^{*} \lambda-\lambda .
$$

We shall omit the subscripts when it does not lead to a confusion.

The proof of the following proposition is straightforward, cf. [5, Theorem 3.1]. 
Proposition 2.1 The map $\mathfrak{G}$ satisfies each of the following conditions:

(1) $\mathfrak{G}$ is a two-cocycle on $\operatorname{Symp}(M, d \lambda)$,

(2) the cohomology class $[\mathfrak{G}] \in H^{2}(\operatorname{Symp}(M, d \lambda) ; \mathbf{R})$ does not depend on the choice of $x \in M$,

(3) the cohomology class $[\mathfrak{G}]$ does not depend of the choice of primitive $\lambda$.

2.A. An alternative approach via a one-cocycle $\quad$ Let $g \in \operatorname{Symp}(M, d \lambda)$. Recall that $g^{*} \lambda-\lambda$ is exact since $g$ preserves $d \lambda$ and $b_{1}(M)$ is assumed to vanish. Therefore there exists a function $\mathcal{K}_{\lambda}(g)$ unique up to a constant (as we have assumed that $M$ is connected) such that

$$
d \mathcal{K}_{\lambda}(g)=g^{*} \lambda-\lambda
$$

The map

$$
\mathcal{K}_{\lambda}: \operatorname{Symp}(M, d \lambda) \rightarrow C^{\infty}(M) / \mathbf{R}
$$

is a one-cocycle on the group of symplectic diffeomorphisms of $(M, d \lambda)$ with values in the right representation of smooth functions on $M$ modulo the constants. The action of a diffeomorphism on a function is by the composition. That is, the map $\mathcal{K}_{\lambda}$ satisfies the following identity

$$
\mathcal{K}_{\lambda}(g h)=\mathcal{K}_{\lambda}(g) \circ h+\mathcal{K}_{\lambda}(h),
$$

which is straightforward to check. This cocycle has been investigated by the authors in [2].

Consider the following short exact sequence of $\operatorname{Symp}(M, d \lambda)$-representations

$$
0 \rightarrow \mathbf{R} \rightarrow C^{\infty}(M) \rightarrow C^{\infty}(M) / \mathbf{R} \rightarrow 0
$$

Proposition 2.3 Consider the connecting homomorphism

$$
\delta: H^{1}\left(\operatorname{Symp}(M, d \lambda), C^{\infty}(M) / \mathbf{R}\right) \rightarrow H^{2}(\operatorname{Symp}(M, d \lambda) ; \mathbf{R}) .
$$

corresponding to the above extension of representations (see Brown [1, III.6] for definition). Then $\delta\left[\mathcal{K}_{\lambda}\right]=[\mathfrak{G}]$.

Proof We start with choosing a lift $\widetilde{\mathcal{K}}_{\lambda}: \operatorname{Symp}(M, d \lambda) \rightarrow C^{\infty}(M)$ of $\mathcal{K}_{\lambda}$. This may be obtained by setting $\widetilde{\mathcal{K}}_{\lambda}(g)(x)=0$. It follows from the identity (2.2) that the coboundary

$$
\delta \widetilde{\mathcal{K}}_{\lambda}(g, h):=\widetilde{\mathcal{K}}_{\lambda}(g) \circ h-\widetilde{\mathcal{K}}_{\lambda}(g h)+\widetilde{\mathcal{K}}_{\lambda}(h)
$$

belongs to $\mathbf{R}$, i.e. it is a constant function. Therefore, without loss of generality, we can evaluate it at point $x$ :

$$
\begin{aligned}
\delta \widetilde{\mathcal{K}}_{\lambda}(g, h)(x) & =\widetilde{\mathcal{K}}_{\lambda}(g)(h x)-\widetilde{\mathcal{K}}_{\lambda}(g h)(x)+\widetilde{\mathcal{K}}_{\lambda}(h)(x) \\
& =\widetilde{\mathcal{K}}_{\lambda}(g)(h x) \\
& =\widetilde{\mathcal{K}}_{\lambda}(g)(h x)-\widetilde{\mathcal{K}}_{\lambda}(g)(x) \\
& =\int_{x}^{h(x)} d \mathcal{K}_{\lambda}
\end{aligned}
$$




$$
\begin{aligned}
& =\int_{x}^{h(x)} g^{*} \lambda-\lambda \\
& =\mathfrak{G}_{x, \lambda}(g, h) .
\end{aligned}
$$

Remark 2.4 Notice that $\mathfrak{G}_{x, \lambda}(g, h)=\mathcal{K}_{\lambda}(g)(h(x))-\mathcal{K}_{\lambda}(g)(x)$.

\section{Proofs of the results}

Let us choose a path $\ell_{x, y}:[0,1] \rightarrow M$ from the basepoint $x \in M$ to a point $y \in M$. Let $-\ell_{x, y}(t):=\ell_{x, y}(1-t)$. Let $\mathfrak{k} \in C^{1}(\operatorname{Symp}(M, d \lambda) ; \mathbf{R})$ be a cochain defined by

$$
\mathfrak{k}(g):=\int_{\ell_{x, g(x)}} \lambda .
$$

Lemma 3.1 Let $\triangle \subset M$ be a trilateral with sides $\ell_{x, g(x)}, g \ell_{x, h x},-\ell_{x, g h x}$. Then

$$
(\mathfrak{G}+\delta \mathfrak{k})(g, h)=\int_{\triangle} d \lambda .
$$

Proof It is the following direct calculation.

$$
\begin{aligned}
(\mathfrak{G}+\delta \mathfrak{k})(g, h) & =\left(\int_{g \ell_{x, h x}} \lambda-\int_{\ell_{x, h x}} \lambda\right)+\left(\int_{\ell_{x, g x}} \lambda-\int_{\ell_{x, g h x}} \lambda+\int_{\ell_{x, h x}} \lambda\right) \\
& =\int_{g \ell_{x, h x}} \lambda+\int_{\ell_{x, g x}} \lambda-\int_{\ell_{x, g h x}} \lambda \\
& =\int_{\Delta} d \lambda .
\end{aligned}
$$

Remark 3.2 Notice that the choice of paths cannot be made continuous if $M$ is not contractible. That is, the path fibration $P M \rightarrow M \times M$ defined by $\ell \mapsto(\ell(0), \ell(1))$ does not admit a continuous section in general.

Proof of Theorem 1.1 Let $M \rightarrow X$ be the universal cover. Consider the composition $X \rightarrow$ $B \pi_{1}(X) \rightarrow B \operatorname{Symp}(M, d \lambda)^{d}$ of the map classifying the universal cover followed by the map induced by the inclusion $\pi_{1}(X) \subset \operatorname{Symp}(M, d \lambda)$ as the deck transformations. The strategy is to show that pullback of the cocycle $\mathfrak{G}$ with respect to this map represents the class $[\sigma]$ of the symplectic form. In fact, we shall prove the following more general result.

Theorem 3.3 Let $(M, d \lambda)$ be a connected regular cover of a closed symplectic $2 n$-manifold $(X, \sigma)$. Let $\Gamma \subset \operatorname{Symp}(M, d \lambda)$ denote the deck transformation group. Suppose that $H^{1}(M ; \mathbf{R})=0$. Then the pullback of the class $[\mathfrak{G}]$ by the homomorphism induced by the composition $X \rightarrow B \Gamma \rightarrow B \operatorname{Symp}(M, d \lambda)^{d}$ is equal to the class represented by the symplectic form $\sigma$. In particular, $[\mathfrak{G}]^{n} \neq 0$. 
Proof The classifying space $B \Gamma$ is constructed as a realisation of a simplicial set according to Milnor $[11,12]$. In this simplicial complex the set of $n$-simplices is identified with $G^{n}$. This gives an identification of the cochain complex for the group cohomology and the simplicial cochain complex (see [16, Section II.1.1.B] for details).

Let us choose a CW-complex structure with a single vertex in $X$. It is always possible due to a standard argument (see for example [3, Proposition 4.2.13] and the subsequent discussion). Such a structure induces a CW-structure on the covering $M$. Notice that the vertices (zero-cells) of this induced structure can be identified with $\Gamma$. Let $x \in M$ be a reference vertex.

With the above choice the classifying map $c: X \rightarrow B \Gamma$ is cellular on the one-skeleton and after an appropriate subdivision of $X$ it can be made cellular on the two-skeleton. Here we consider the simplicial structure on $B \Gamma$ as a $\mathrm{CW}$-complex.

Let $\Delta$ be an oriented two-cell of $X$. Its image $c(\Delta)$ with respect to the classifying map is a two-simplex in $B \Gamma$ and hence a pair of elements from $\Gamma$. To find these elements consider the lift of $\Delta$ to $M$ passing through the reference vertex $x$. Let $\Delta_{x}$ denote this lift. The vertices of $\Delta_{x}$ are of the form $x, g x, g h x$ for some $g, h \in \Gamma$. Thus $c(\Delta)$ is identified with the pair $(g, h) \in \Gamma \times \Gamma$.

Let us represent the cohomology class [ $\mathfrak{G}]$ by the cocycle $\mathfrak{G}+\delta \mathfrak{k}$ as in Lemma 3.1. Then we pull it back to $\Gamma$ and consider it as a CW-cocycle, pull it back to $X$ and evaluate on a two-cell $\Delta$.

$$
\begin{aligned}
\left\langle c^{*}(\mathfrak{G}+\delta \mathfrak{k}), \Delta\right\rangle & =\langle\mathfrak{G}+\delta \mathfrak{k}, c(\Delta)\rangle \\
& =(\mathfrak{G}+\delta \mathfrak{k})(g, h) \\
& =\int_{\Delta_{x}} d \lambda \\
& =\int_{\Delta} \sigma
\end{aligned}
$$

That is, the pull back of the cocycle $\mathfrak{G}+\delta \mathfrak{k}$ to $X$ is a cocycle defined by the integration of the symplectic form $\sigma$. Thus it represents the $\mathrm{CW}$-cohomology class corresponding to the cohomology class of $\sigma$ under the de Rham isomorphism. In particular, since $[\sigma]^{n} \neq 0$, we get that $[\mathfrak{G}]^{n} \neq 0$.

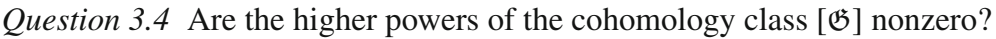

Proof of Theorem 1.5 ( $c f$. [5, Section 4.2]) Recall that we need to show that the pullback of the cocycle $\mathfrak{G}$ with respect to the inclusion $G=\operatorname{Isom}^{\circ}(M) \subset \operatorname{Symp}(M, d \lambda)$ is cohomologous to the Kähler cocycle $\mathfrak{K}$. It immediately follows from Lemma 3.1.

As we pointed out in the introduction a stronger statement is true.

Proposition 3.5 Let $x \in M$ be a reference point. There exists a primitive $\lambda$ such that the cocycle $\mathfrak{G}_{x, \lambda}$ is equal to the Kähler cocycle $\mathfrak{K}$.

Proof A primitive $\lambda$ can be written as $\lambda=-J d \varphi$ where $J$ is the complex structure on $M$. The function $\varphi$ is called the Kähler potential. Averaging $\varphi$ with respect to the (compact) stabiliser of a point $x$, one can choose $\varphi$ to be radial (as the stabiliser of $x$ in $G$ acts transitively on the unit tangent sphere at $x)$, i.e. $\varphi=u(\operatorname{dist}(\cdot, x))$ for a suitable function $u:[0, \infty) \rightarrow \mathbf{R}$.

Let $L$ be the Liouville vector field defined by $i_{L} d \lambda=\lambda$. By definition of $\lambda$, the vector field $L$ is the metric gradient of the function $\varphi$. Recall that the spheres around $x$ (the level sets 
of the function $\varphi$ ) are orthogonal to the geodesics from $x$. Therefore the flow of $-L$ contracts $M$ to the unique zero $x$ of $L$ along the geodesics. Let $\ell_{x, y}$ denote the geodesic between $x$ and $y$.

A flow line of the Liouville vector field $L$ is $\lambda$-null, as $i_{L} \lambda=\left(i_{L}\right)^{2} d \lambda=0$. Thus $\int_{\ell_{x, y}} \lambda=0$, for every $y$. Therefore

$$
\mathfrak{K}(g, h)=\int_{\ell_{x, g x}} \lambda+\int_{\ell_{g x, g h x}} \lambda-\int_{\ell_{x, g h x}} \lambda=\int_{\ell_{g x, g h x}} \lambda,
$$

and

$$
\mathfrak{G}_{x, \lambda}(g, h)=\int_{\ell_{g x, g h x}} \lambda-\int_{\ell_{x, h x}} \lambda=\int_{\ell_{g x, g h x}} \lambda .
$$

Example 3.6 If $M=\mathrm{U}(1,1) / \mathrm{SO}(2)$ is a complex hyperbolic line then the function $u:[0, \infty) \rightarrow \mathbf{R}$ from the first paragraph of the above proof is defined by $u(r)=$ $\log (\cosh (r)+1)$.

Question 3.7 What are the maximal subgroups of $\operatorname{Symp}(M, d \lambda)$ on which the cocycle $\mathfrak{G}$ is cohomologous to a bounded one?

Proof of Theorem 1.2 Observe that $g^{*} \lambda-\lambda$ vanishes outside the support of $g$. Therefore if $g$ has a compact support, one may try to normalise $\mathcal{K}(g)$ to vanish outside the support of $g$ as well. However in [2, p. 78] we construct an example (with $M=T^{\vee} S^{1}$, the cotangent bundle of $\left.S^{1}\right)$ where $\mathcal{K}(g)$ takes different values on both ends of $M$. Nevertheless, one can fix an end of $M$ and declare $\mathcal{K}(g)$ to vanish there. This provides a lift of $\mathcal{K}$ to functions on $M$ (without constant ambiguity). Thus the connecting homomorphism sends $\mathcal{K}$ to zero (cf. Proposition 2.3).

Remark 3.8 We have the following alternative argument. Since $M$, being a manifold, is $\sigma$-compact, there exists a ray $\gamma:[0, \infty) \rightarrow M$ starting at $x$ and leaving any compact subset of $M$. For $g \in \operatorname{Symp}_{c}(M, d \lambda)$ define $\mathfrak{b}(g):=\int_{\gamma} g^{*} \lambda-\lambda$. Notice that this makes sense as eventually, along $\gamma$, outside the support of $g$ one has $g^{*} \lambda=\lambda$. We have the following computation in which a curve from $x$ to $h(x)$ is chosen to be the concatenation of a part of $\gamma$ from $x$ to the outside of the union of the supports of $g$ and $h$ and then the part of $-h(\gamma)$ back to $h(x)$.

$$
\begin{aligned}
\mathfrak{G}_{x, \lambda}(g, h) & =\int_{x}^{h(x)} g^{*} \lambda-\lambda \\
& =\int_{\gamma} g^{*} \lambda-\lambda-\int_{h(\gamma)} g^{*} \lambda-\lambda \\
& =\int_{\gamma} g^{*} \lambda-\lambda-\int_{\gamma}(g h)^{*} \lambda-\lambda+\int_{\gamma} h^{*} \lambda-\lambda=\delta \mathfrak{b}(g, h)
\end{aligned}
$$


Proof of Theorem 1.3 Recall that $L$ is an exact isotropic submanifold of $M$. Assume that $i^{*} \lambda=0$ and choose $x \in L$. Then

$$
\mathfrak{G}_{x . \lambda}(g, h)=\int_{x}^{h(x)} g^{*} \lambda-\lambda=0,
$$

since the curve joining $x$ and $h(x)$ can be chosen to be contained in $L$ and $i^{*}\left(g^{*} \lambda\right)=$ $g^{*}\left(i^{*} \lambda\right)=0$.

Observe that we can always find a primitive $\lambda$ such that $i^{*} \lambda=0$. Indeed, let $\lambda^{\prime}$ be a primitive with the property that $\left[i^{*} \lambda^{\prime}\right]=0$. We have $i^{*}\left(\lambda^{\prime}\right)=d F^{\prime}$ for some function $F^{\prime}: L \rightarrow \mathbf{R}$. Extending $F^{\prime}$ to a function $F: M \rightarrow \mathbf{R}$ we obtain $i^{*}\left(\lambda^{\prime}-d F\right)=0$ and we take $\lambda:=\lambda^{\prime}-d F$.

Example 3.9 The cohomology class [G] vanishes on the following subgroups of $\operatorname{Symp}(M, d \lambda)$ :

(1) $\operatorname{Symp}(M, x)$ - the isotropy of a point $x \in M$;

(2) $\operatorname{Diff}(L) \subset \operatorname{Symp}\left(T^{\vee} L\right)$ where $M=T^{\vee} L$ is the cotangent bundle of $L$.

Example 3.10 Let $(M, d \lambda)$ be the universal cover of $(X, \sigma)$. The deck transformation group $\pi_{1}(X) \subset \operatorname{Symp}(M, d \lambda)$ preserves the orbit of $x \in M$. Such an orbit is clearly isotropic. This shows that the connectivity of $L$ is essential for Theorem 1.3 to hold, according to Theorem 1.1.

Let $S \subset \pi_{1}(X)$ be a finite set of generators. The associated Cayley graph $\Gamma_{S}$ can be embedded in $M$ as a connected isotropic subspace invariant under the deck transformations. To do this consider the map from a wedge of circles $Y$, one per generator of $\pi_{1}(X)$ and map it into $X$. Then the Cayley graph $\Gamma_{S}$ is a covering of $Y$ and the map lifts to the equivariant map into $M$. The primitive $\lambda$ represents a nontrivial cohomology class of $\Gamma_{S}$.

For example, let $\Sigma$ be a surface of positive genus $g$. Then $\Gamma_{S}$ can be arranged to be a tesselation of a plane with $4 g$-gons. The integral of $\lambda$ over the boundary of a sigle polygon is, by the Stokes lemma, equal to its area and hence nonzero. This shows that the hypothesis that $\left[i^{*} \lambda\right]=0$ is also essential.

In this example the isotropic subspace is not a submanifold but it can be improved by taking a surface of genus equal to the number of generators of $G$ and mapping it as an isotropic subset into $X$. This can be done provided the dimension of $X$ is big enough. The lift to $M$ is a $\pi_{1}(X)$-invariant closed isotropic submanifold of $M$.

Proof of Theorem 1.4 Let us explain first that if $(X, \sigma)$ is a closed symplectic manifold with an exact universal cover $(M, d \lambda)$ then there is an injective homomorphism $\operatorname{Ham}(X, \sigma) \rightarrow$ $\operatorname{Symp}(M, d \lambda)$.

Let $f_{t} \in \operatorname{Ham}(X, \sigma)$ be an isotopy from the identity to $f=f_{1}$. This isotopy can be lifted to an isotopy $\tilde{f}_{t} \in \operatorname{Symp}(M, d \lambda)$ from the identity to $\tilde{f}=\tilde{f}_{1}$. Since the evaluation map $\operatorname{Ham}(X, \sigma) \rightarrow X$ induces the trivial homomorphism on the fundamental group [10, Corollary 9.1.2], the endpoint $\tilde{f}$ does not depend on the choice of the isotopy $f_{t}$.

We shall prove that $\mathfrak{G}$ restricted to $\operatorname{Ham}(X, \sigma)$ is a coboundary. Recall that, due to Proposition 2.3, $[\mathfrak{G}]=\delta\left[\mathcal{K}_{\lambda}\right]$. We shall show that the restriction of the cocycle $\mathcal{K}_{\lambda}$ to $\operatorname{Ham}(X, \sigma)$ admits a lift to a cocycle $\widetilde{\mathcal{K}}_{\lambda}: \operatorname{Ham}(X, \sigma) \rightarrow C^{\infty}(M)$.

Let $f_{t} \in \operatorname{Ham}(X, \sigma)$, for $t \in[0,1]$ be a Hamiltonian isotopy from the identity to $f=f_{1}$ generated by a normalised Hamiltonian function $H_{t}: X \rightarrow \mathbf{R}$. Recall that $H_{t}$ is normalised 
if $\int_{X} H_{t} \sigma^{n}=0$ for all $t \in[0,1]$. Let $F_{t}: M \rightarrow \mathbf{R}$ be defined by

$$
F_{t}(x)=\int_{0}^{t}\left(\lambda\left(X_{s}\right)+\widetilde{H}_{s}\right)\left(\tilde{f}_{s}(x)\right) d s .
$$

Here $\widetilde{H}_{t}$ is the lift of the Hamiltonian $H_{t}$ and $X_{t}$ is the corresponding vector field. According to [2, Proposition 2.8] (cf. [9, Proposition 9.19]; beware that [9] uses the opposite sign convention for Hamiltonians) we have

$$
d F_{t}=d \mathcal{K}_{\lambda}\left(\tilde{f}_{t}\right)
$$

Let $\widetilde{\mathcal{K}}_{\lambda}(f):=F_{1}$. We need to check that this definition does not depend on the choice of isotopy from the identity to $f$. Let $\left\{f_{t}\right\}$ and $\left\{f_{t}^{\prime}\right\}$ be two Hamiltonian isotopies from the identity to $f \in \operatorname{Ham}(X, \sigma)$. Let $\left\{\tilde{f}_{t}\right\}$ and $\left\{\tilde{f}_{t}^{\prime}\right\}$ denote their lifts to $\operatorname{Ham}(M, d \lambda)$. As explained above $\tilde{f}_{1}=\tilde{f}_{1}^{\prime}$. The formula 3.11 defines two time-dependent functions $F_{t}$ and $F_{t}^{\prime}$. Observe that the difference $F_{1}-F_{1}^{\prime}$ is constant because

$$
d\left(F_{1}-F_{1}^{\prime}\right)=d\left(\mathcal{K}_{\lambda}\left(\tilde{f}_{1}\right)-\mathcal{K}_{\lambda}\left(\tilde{f}_{1}^{\prime}\right)\right) .
$$

The following calculation shows that this constant is equal to the function corresponding to the concatenation of the isotopy $\left\{f_{t}\right\}$ and the isotopy $\left\{f_{1-t}^{\prime}\right\}$. Let $g:[0,2] \rightarrow \operatorname{Ham}(X, \sigma)$ denote this concatenation and let $G$ and $Y$ denote its Hamiltonian function and the generated vector field respectively.

$$
\begin{aligned}
& \int_{0}^{2}\left(\lambda\left(Y_{s}\right)+\widetilde{G}_{s}\right)\left(\tilde{g}_{s}(x)\right) d s \\
& =\int_{0}^{1}\left(\lambda\left(Y_{s}\right)+\widetilde{G}_{s}\right)\left(\tilde{g}_{s}(x)\right) d s+\int_{1}^{2}\left(\lambda\left(Y_{s}\right)+\widetilde{G}_{s}\right)\left(\tilde{g}_{s}(x)\right) d s \\
& =\int_{0}^{1}\left(\lambda\left(Y_{s}\right)+\widetilde{G}_{s}\right)\left(\tilde{g}_{s}(x)\right) d s+\int_{0}^{1}\left(\lambda\left(Y_{2-t}\right)+\widetilde{G}_{2-t}\right)\left(\tilde{g}_{2-t}(x)\right) d t \\
& =\int_{0}^{1}\left(\lambda\left(X_{s}\right)+\widetilde{H}_{s}\right)\left(\tilde{f}_{s}(x)\right) d s-\int_{0}^{1}\left(\lambda\left(X_{t}^{\prime}\right)+\widetilde{H}_{t}^{\prime}\right)\left(\tilde{f}_{t}^{\prime}(x)\right) d t \\
& =F_{1}-F_{1}^{\prime}
\end{aligned}
$$

Consequently, the proof is reduced to showing that if $\left\{f_{t}\right\}$ is a loop in $\operatorname{Ham}(X, \sigma)$ based at the identity then $F_{1}(x)=0$ for all $x \in M$. We have that

$$
F_{1}(x)=\int_{\tilde{f}_{t}(x)} \lambda+\int_{0}^{1} \widetilde{H}_{t}\left(\tilde{f}_{t}(x)\right) d t .
$$

and this quantity is known as the action functional of the Hamiltonian loop $\left\{f_{t}\right\}$. According to Schwarz [15, Lemma 3.3], $F_{1}$ is constant and depends only on the homotopy class of the loop $\left\{f_{t}(x)\right\}$. Finally, it follows from the proof of Proposition 3.1 (i) in McDuff [8, page 311] that $F_{1}(x)$ is equal to zero. This finishes the proof of Theorem 1.4. 
It is important that we ask about vanishing of the cocycle on the group of Hamiltonian diffeomorphisms of a compact quotient of exact symplectic manifold $M$, i.e. the group generated by periodic (with respect to the action of the deck-transformations group $\Gamma$ ) Hamiltonians. We already know that the cocycle is nontrivial on $\operatorname{Ham}(M)$ when $M$ is a symmetric space of Hermitian type. This motivates the following question.

Question 3.13 Does $\mathfrak{G}$ vanish on the group generated by bounded Hamiltonians on $M$ ? Given a complete Riemannian metric on $M$, does $\mathfrak{G}$ vanish on the group generated by Hamiltonians with bounded differential?

\section{Boundedness properties of $\mathfrak{G}$}

Let $\mathfrak{c}$ be a real valued two-cocycle on a group $G$. An element $g$ of $G$ defines a function $g_{\mathfrak{c}: ~} \rightarrow \mathbf{R}$ by the formula

$$
{ }^{g} \mathfrak{c}(h)=\mathfrak{c}(g, h) .
$$

We say that $\mathfrak{c}$ is semibounded if ${ }^{g} \mathfrak{c}$ is a bounded function on $G$ for any $g \in G$. By $|g|_{\mathfrak{c}}$ we denote the supremum norm of ${ }^{g} \mathrm{c}$ :

$$
|g|_{\mathfrak{c}}:=\sup _{h \in G}|\mathfrak{c}(g, h)| .
$$

Lemma 4.1 Assume that $\mathfrak{c}$ is a semibounded two-cocycle on $G$. Then for all $f, g \in G$

$$
|f g|_{\mathfrak{c}} \leq 2|f|_{\mathfrak{c}}+|g|_{\mathfrak{c}}
$$

Proof By the cocycle identity

$$
\begin{aligned}
|f g|_{\mathfrak{c}} & =\sup _{h}|\mathfrak{c}(f g, h)| \\
& \leq \sup _{h}(|\mathfrak{c}(f, g)|+|\mathfrak{c}(f, g h)|+|\mathfrak{c}(g, h)|) \\
& \leq 2 \sup _{h}|\mathfrak{c}(f, h)|+\sup _{h}|\mathfrak{c}(g, h)| . \\
& =2|f|_{\mathfrak{c}}+|g|_{\mathfrak{c}} .
\end{aligned}
$$

A closed symplectic manifold $(X, \sigma)$ is called symplectically hyperbolic if the pullback of the symplectic form $\sigma$ to the universal cover is exact and admits a primitive that is bounded with respect to the Riemannian metric induced from an auxiliary metric on $X$ [14, Definition 1.2.C]. Examples and constructions of such manifolds are discussed in [6].

Proposition 4.2 Let $(X, \sigma)$ be a symplectically hyperbolic manifold and let $(M, d \lambda)$ be its universal cover. Then $\mathfrak{G}$ is a semibounded cocycle on $\operatorname{Ham}(X, \sigma)$.

Proof Let $g, h \in \operatorname{Ham}(X, \sigma)$ be generated by isotopies $g_{t}$ and $h_{t}$ respectively, with the corresponding Hamiltonian functions $G_{t}$ and $H_{t}$. Let $\tilde{g}_{t}, \tilde{h}_{t}$ and $\widetilde{G}_{t}, \widetilde{H}_{t}$ be the lifts to $M$. We need to prove that

$$
\sup _{h \in \operatorname{Ham}(X, \sigma)} \mathfrak{G}(\tilde{g}, \tilde{h})
$$

is finite. 
Recall from Remark 2.4 that $\mathfrak{G}(\tilde{g}, \tilde{h})=\mathcal{K}_{\lambda}(\tilde{g})(\tilde{h}(x))-\mathcal{K}_{\lambda}(\tilde{g})(x)$. Thus the statement will follow from the boundedness of $\mathcal{K}_{\lambda}(\tilde{g})$ which was proven in [2, Proposition 6.1]. We recall the proof here for the convenience of the reader.

Let $x, y \in M$ and let $C>0$ be a constant bounding the one-form $\lambda$ on $M$ with respect to a Riemannian metric induced from a metric on $X$. The first equality in the following calculation follows from the formulae in the proof of Theorem 1.4 expressing $\mathcal{K}_{\lambda}$ in terms of the action functional.

$$
\begin{aligned}
& \left|\mathcal{K}_{\lambda}(\tilde{g})(y)-\mathcal{K}_{\lambda}(\tilde{g})(x)\right| \\
& \quad=\left|\int_{\tilde{g}_{t}(y)} \lambda+\int_{0}^{1} \widetilde{G}_{t}\left(\tilde{g}_{t}(y)\right) d t-\int_{\tilde{g}_{t}(x)} \lambda+\int_{0}^{1} \widetilde{G}_{t}\left(\tilde{g}_{t}(x)\right) d t\right| \\
& \quad \leq 2 C \max _{x} \operatorname{Length}\left(\tilde{g}_{t}(x)\right)+2 \max _{x, t} \widetilde{G}_{t}(x)<\infty
\end{aligned}
$$

The last quantity is finite because $\widetilde{G}_{t}$ and $\tilde{g}_{t}$ are lifts of $G_{t}$ and $g_{t}$ respectively and the latter are defined on a compact manifold $X$. Also, the length is calculated with respect to the metric induced from $X$. We also used a straightforward fact that $\int_{f_{t}(x)} \lambda \leq C \operatorname{Length}\left(f_{t}(x)\right)$.

Remark 4.3 The above also shows that if $|g|_{\mathfrak{G}}=0$ then $\mathcal{K}_{\lambda}(g)$ is constant and therefore $g^{*} \lambda=\lambda$ which cannot happen if $g \in \operatorname{Ham}(X, \sigma)$. The reason why $\mathcal{K}_{\lambda}(g)$ cannot be constant is explained in [2, Theorem 4.1 (1)]. Namely it follows from Schwarz's result that such $g$ has two fixed points on which the action functional (see formula 3.12) defining $\mathcal{K}_{\lambda}(g)$ assumes different values.

Let $\Gamma$ be a finitely generated group. Let $|g|_{S}$ denote the word length of an element $g$ of $\Gamma$ with respect to a fixed finite set of generators $S$.

Proposition 4.4 Let $\mathfrak{c}$ be a semibounded cocycle on $\Gamma$ then $|\cdot|_{\mathfrak{c}}$ is Lipschitz with respect to the word-length. More precisely

$$
|g|_{\mathfrak{c}} \leq\left(2 \max _{s \in S}|s|_{\mathfrak{c}}\right)|g|_{S}
$$

Proof Let $s$ be one of the generators. By Lemma 4.1 we have

$$
|s g|_{\mathfrak{c}} \leq 2|s|_{\mathfrak{c}}+|g|_{\mathfrak{c}} .
$$

Then, by induction,

$$
|g|_{\mathfrak{c}}=\left|s_{i_{1}} \ldots s_{i_{|g| S}}\right|_{\mathfrak{c}} \leq 2\left|s_{i_{1}}\right|_{\mathfrak{c}}+\cdots+2\left|s_{i_{|g| S}}\right|_{\mathfrak{c}} \leq 2\left(\max _{s \in S}|s|_{\mathfrak{c}}\right)|g|_{S} .
$$

On the other hand, as we shall explain next, the behaviour of $\mathfrak{G}$ with respect to the first argument is very different. Let

$$
\operatorname{Symp}(M, x, y):=\{f \mid f(x)=x \text { and } f(y)=y\}
$$

be the subgroup consisting of symplectic diffeomorphisms preserving the points $x, y \in M$. Let $h \in \operatorname{Symp}(M, d \lambda)$. Define

$$
\mathfrak{G}_{x}^{h}: \operatorname{Symp}(M, x, h(x)) \rightarrow \mathbf{R}
$$


by $\mathfrak{G}_{x}^{h}(f):=\mathfrak{G}_{x, \lambda}(f, h)$ and observe that it is a homomorphism of groups.

$$
\begin{aligned}
\mathfrak{G}_{x}^{h}(f g) & =\int_{x}^{h(x)} g^{*} f^{*} \lambda-\lambda \\
& =\int_{x}^{h(x)} g^{*} f^{*} \lambda-g^{*} \lambda+g^{*} \lambda-\lambda \\
& =\int_{g(x)}^{g h(x)} f^{*} \lambda-\lambda+\int_{x}^{h(x)} g^{*} \lambda-\lambda \\
& =\int_{x}^{h(x)} f^{*} \lambda-\lambda+\int_{x}^{h(x)} g^{*} \lambda-\lambda \\
& =\mathfrak{G}_{x}^{h}(f)+\mathfrak{G}_{x}^{h}(g)
\end{aligned}
$$

It follows from the Stokes Lemma that $\mathfrak{G}_{x}^{h}(g)$ is equal to the symplectic area of a disc bounded by $g(\gamma)-\gamma$ where $\gamma$ is a curve from $x$ to $h(x)$. Hence it is straightforward to show, by a local construction in a Darboux chart, that if $h(x) \neq x$ then the homomorphism $\mathfrak{G}_{x}^{h}$ is nontrivial.

Proof of Theorem 1.6 The above argument proves that the cocycle $\mathfrak{G}_{x}$ is unbounded. Observe, e.g. by a local construction mentioned above, that it directly applies to the sub$\operatorname{group} \operatorname{Ham}(X, \sigma) \subset \operatorname{Symp}(M, d \lambda)$ if $(M, d \lambda)$ is the universal cover of $(X, \sigma)$.

\section{Applications}

5.A. Symplectic actions of finitely generated groups

For an element $g$ of a finitely generated group $\Gamma$ one defines its translation length as

$$
\|g\|:=\lim _{n \rightarrow \infty} \frac{\left|g^{n}\right| S}{n},
$$

where $|g|_{S}$ denotes the word length of an element $g$ of $\Gamma$ with respect to a fixed finite set of generators $S$.

Remark 5.1 Another terminology says that the cyclic subgroup generated by $g$ is undistorted in $G$ if the translation length of $g$ does not vanish. Observe that the (non-) vanishing of the translation length does not depend on the choice of generators.

Theorem 5.2 (Polterovich [14, Theorem 1.6.A]) Let $(X, \sigma)$ be a closed symplectically hyperbolic manifold. If $\Gamma \subset \operatorname{Ham}(X, \sigma)$ is a finitely generated group then every nontrivial element of $\Gamma$ has nonzero translation length.

Proof Fix a nontrivial element $g$ in $\Gamma \subset \operatorname{Ham}(X, \sigma)$. According to a theorem of Schwarz [15] (see also Theorem 9.1.6 in [10]) $g$ has two contractible fixed points $x, y \in X$ with nonzero action difference.

Choose $h \in \operatorname{Ham}(X, \sigma)$ such that $h(x)=y$. Then

$$
\mathfrak{G}(g, h)=\mathcal{K}_{\lambda}(g)(h(x))-\mathcal{K}_{\lambda}(g)(x) \neq 0
$$

since this is equal to the action difference as explained in [2, Lemma 3.4] and [14, Section 2.1]. Then

$$
2 \max _{s \in S}|s|_{\mathfrak{G}} \frac{\left|g^{n}\right|_{S}}{n} \geq \frac{\left|g^{n}\right| \mathfrak{G}}{n} \geq \frac{\left|\mathfrak{G}\left(g^{n}, h\right)\right|}{n}=|\mathfrak{G}(g, h)|,
$$


where the first inequality follows from Proposition 4.2 and Proposition 4.4, the second from the very definition of $|\cdot|_{\mathfrak{G}}$ and the last equality from the fact that $\mathfrak{G}_{x}^{h}$ is a homomorphism. Therefore

$$
\|g\|=\lim _{n \rightarrow \infty} \frac{\left|g^{n}\right|_{S}}{n} \geq \frac{|\mathfrak{G}(g, h)|}{2 \max _{s \in S}|s|_{\mathfrak{G}}}>0 .
$$

Remark 5.3 We gave a similar proof of this theorem in [2]. The new element in the above proof is the use of the semiboundedness property of the cocycle $\mathfrak{G}$.

5.B. Foliated symplectic bundles Recall that the cohomology of a group $G$ is isomorphic to the cohomology of the classifying space $B G^{d}$. Thus the cohomology class $[\mathfrak{G}]$ is a characteristic class for symplectic foliated bundles (see for example Section 2.3.3 in Morita [13]). By this we mean a bundle $(M, d \lambda) \rightarrow E \rightarrow B$ admitting a foliation transverse to the fibres and such that its holonomy is a discrete subgroup of $\operatorname{Symp}(M, d \lambda)$. The corresponding characteristic class in $H^{2}(B ; \mathbf{R})$ will be denoted by $\mathfrak{G}(E)$.

We say that a bundle $L \rightarrow E^{\prime} \rightarrow B$ is a foliated subbundle of $E$ if it is a subbundle and the total space $E^{\prime}$ is a union of the leaves of the foliation in $E$. Existence of such a subbundle is equivalent to the reduction of the structure group from $\operatorname{Symp}(M, d \lambda)^{d}$ to a subgroup preserving the subspace $L \subset M$. Here $L$ and $M$ are identified with the fibres over $b \in B$ of $E^{\prime}$ and $E$ respectively. The following result is a direct consequence of Theorem 1.3.

Corollary 5.4 Let $i: L \rightarrow M$ be the inclusion of an exact isotropic submanifold. Let $(M, d \lambda) \rightarrow E \rightarrow B$ be a foliated symplectic bundle. If it admits a foliated subbundle $L \rightarrow E^{\prime} \rightarrow B$ then the cohomology class $\mathfrak{G}(E) \in H^{2}(B ; \mathbf{R})$ is trivial.

The above corollary gives an obstruction to the existence of foliated subbundles with isotropic fibres. The next result, that immediately follows from Theorem 1.1 (and its proof), provides a construction of foliated symplectic bundles with a nontrivial obstruction.

Corollary 5.5 Let $(M, d \lambda)$ be the universal cover of a closed symplectic $2 n$-manifold $(X, \sigma)$. The flat bundle

$$
M \rightarrow E:=M \times_{\pi_{1}(X)} M \rightarrow X
$$

has nontrivial characteristic class $\mathfrak{G}(E)$. Moreover, the class is equal to the cohomology class of the symplectic form and hence $\mathfrak{G}(E)^{n} \neq 0$.

Example 5.6 (1) Let $(M, d \lambda) \rightarrow E \rightarrow B$ is a foliated symplectic bundle admitting a section whose image is equal to a leaf of the foliation then $\mathfrak{G}(E)=0$. Moreover, since the obstruction $\mathfrak{G}(E)$ is a real cohomology class it is zero if the bundle admits a leaf finitely covering the base. Indeed, by pulling-back the bundle over a connected component of such a leaf we obtain a bundle with a section. Moreover, a finite connected covering induces an isomorphism on the real cohomology.

(2) Let $X \rightarrow E^{\prime} \rightarrow B$ be a smooth foliated bundle. Consider vertical cotangent bundle $T^{\vee} X \rightarrow E \rightarrow B$. Observe that the later is flat symplectic bundle and $E^{\prime}$ is a foliated subbundle of $E$. Since the image of the zero section $X \subset T^{\vee} X$ is a Lagrangian submanifold we get $\mathfrak{G}(E)=0$.

(3) Let $\Sigma$ be a closed and oriented surface of positive genus. The foliated bundle $\widetilde{\Sigma} \rightarrow$ $E:=\widetilde{\Sigma} \times_{\pi_{1}(\Sigma)} \widetilde{\Sigma} \rightarrow \Sigma$ does not admit a foliated subbundle of positive codimension. Indeed, it follows from Corollary 5.5 that $\mathfrak{G}(E) \neq 0$. 
(4) The identity map $\operatorname{Symp}(M, d \lambda)^{d} \rightarrow \operatorname{Symp}(M, d \lambda)$ induces a homomorphism $H^{*}(B \operatorname{Symp}(M, d \lambda) ; \mathbf{R}) \rightarrow H^{*}\left(B \operatorname{Symp}(M, d \lambda)^{d} ; \mathbf{R}\right)$. In general, $\mathfrak{G}$ is not contained in the image of this homomorphism. To see this consider $M=\mathbf{R}^{2 n}$. It follows from Theorem 3.3 that the restriction of $\mathfrak{G}$ is nontrivial on $\mathbf{Z}^{2 n} \subset \operatorname{Symp}\left(\mathbf{R}^{2 n}, d \lambda\right)$. However, the composition $\mathbf{Z}^{n} \rightarrow \operatorname{Symp}\left(\mathbf{R}^{2 n}, d \lambda\right)$ factors through the contractible group $\mathbf{R}^{2 n}$ and hence it induces the trivial map on cohomology.

Acknowledgments We warmly thank Dusa McDuff for explaining to us the proof of Theorem 1.4 and Example 5.6 (4). We thank Dieter Kotschick for drawing our attention to the paper of Ismagilov, Losik, and Michor. And, last but not least, the anonymous referee for helpful remarks improving the final exposition.

Open Access This article is distributed under the terms of the Creative Commons Attribution Noncommercial License which permits any noncommercial use, distribution, and reproduction in any medium, provided the original author(s) and source are credited.

\section{References}

1. Brown, K.S.: Cohomology of groups. Graduate Texts in Mathematics, vol. 87. Springer-Verlag, New York (1982)

2. Gal, Ś.R., Kędra, J.: A cocycle on the group of symplectic diffeomorphisms. Adv. Geom. 11(1), 73-88 (2011)

3. Gompf, R.E., Stipsicz, A.I.: 4-manifolds and Kirby calculus. Graduate Studies in Mathematics, vol. 20. American Mathematical Society, Providence (1999)

4. Guichardet, A., Wigner, D.: Sur la cohomologie réelle des groupes de Lie simples réels. Ann. Sci. École Norm. Sup. (4) 11(2), 277-292 (1978)

5. Ismagilov R.S., Losik M., Michor P.W.: A 2-cocycle on a symplectomorphism group. Mosc. Math. J. 6(2), 307-315, 407 (2006)

6. Kędra, J.: Symplectically hyperbolic manifolds. Differential Geom. Appl. 27(4), 455-463 (2009)

7. Kędra, J., Rudyak, Y., Tralle, A.: Symplectically aspherical manifolds. J. Fixed Point Theory Appl. 3(1), 1-21 (2008)

8. McDuff, D.: Monodromy in Hamiltonian Floer theory. Comment. Math. Helv. 85(1), 95-133 (2010)

9. McDuff, D., Salamon, D.: Introduction to symplectic topology, 2nd edn. Oxford Mathematical Monographs. The Clarendon Press Oxford University Press, New York (1998)

10. McDuff, D., Salamon, D.: $J$-holomorphic curves and symplectic topology. American Mathematical Society Colloquium Publications, vol. 2. American Mathematical Society, Providence, RI (2004)

11. Milnor, J.: Construction of universal bundles. I. Ann. Math. 63 (2) , 272-284 (1956)

12. Milnor, J.: Construction of universal bundles. II. Ann. Math. 63 (2) , 430-436 (1956)

13. Morita, S.: Geometry of characteristic class. Translations of Mathematical Monographs, vol. 199. American Mathematical Society, Providence, RI, 2001. Translated from the 1999 Japanese original, Iwanami Series in Modern Mathematics

14. Polterovich, L.: Growth of maps, distortion in groups and symplectic geometry. Invent. Math. 150(3), 655-686 (2002)

15. Schwarz, M.: On the action spectrum for closed symplectically aspherical manifolds. Pac. J. Math. 193(2), 419-461 (2000)

16. Vinberg, E. B. (ed.): Lie Groups and Lie Algebras. II, of Encyclopaedia of Mathematical Sciences, vol. 21. Springer, Berlin (2000). Discrete Subgroups of Lie Groups and Cohomologies of Lie Groups and Lie Algebras, A translation of Current problems in mathematics. Fundamental directions, vol. 21 (Russian), Akad. Nauk SSSR, Vsesoyuz. Inst. Nauchn. i Tekhn. Inform. (VINITI), Moscow, 1988 [MR 89f:22001], Translated by John Danskin, Translation edited by Onishchik A. L., Vinberg E. B.

17. Wienhard, A.K.: Bounded Cohomology and Geometry. Bonner Mathematische Schriften [Bonn Mathematical Publications], 368. Universität Bonn Mathematisches Institut, Bonn, 2004. Dissertation, Rheinische Friedrich-Wilhelms-Universität Bonn, Bonn (2004) 